\title{
Software review: Using short message services as a marketing tool
}

Received: 6th December, 2000

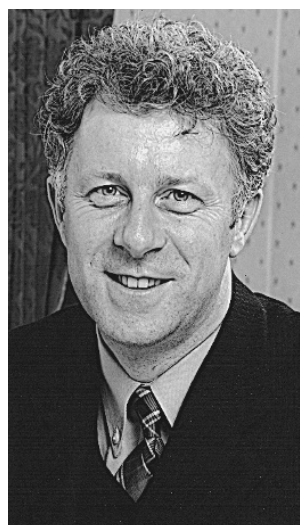

\section{Shaun Doyle}

is Chairman of Intrinsic, a campaign management software company. The focus of the business is the provision of software and database marketing consultancy services aimed at helping clients manage their customer communications more intelligently and effectively. A co-founder of the company with Steve Treadwell, he is responsible for management of the business consultancy team and the strategic direction of the company. He has extensive experience in the design, development, implementation and exploitation of customer-focused database marketing systems in a number of business sectors. These include financial services, retail, leisure, charity and telecommunications. He has been involved in the design and building of over 50 marketing databases in blue-chip organisations.

$\mathrm{He}$ is well known in the industry, regularly speaking and running specialist workshops at leading industry conferences. He has had a number of papers published in the UK and overseas on the application of database marketing.

Abstract The year 2000 saw an explosion in the volume of short text messages being sent to mobile phones. Originally the sole realm of the telecommunication providers, this communication medium is starting to be used by other types of organisation to deliver messages to consumers. Marketers are starting to recognise the potential of this medium for marketing communications. This paper explains how the technology works and explores potential business applications in marketing.

\section{INTRODUCTION}

To date much of the discussion around the wireless Internet has concentrated on the application of Wireless Application Protocol (WAP) technologies. But a survey by Forrester Research ${ }^{1}$ showed that many WAP sites have a long way to go in meeting the requirements of the consumer for reliability, access and navigation. Short message services (SMS) available on GSM mobile phones on the other hand have grown from strength to strength. Unlike WAP, SMS can be used as a two-way communication vehicle, allowing people or organisations to send and receive short text messages from a mobile phone in near real time. The ready uptake by consumers of SMS will ensure that it becomes an integral part of the marketing mix.

\section{THE TECHNOLOGY BEHIND SMS}

The SMS service is actually a network of SMS centres that are connected to each other and can interchange text-based messages. The SMS centres are specially written software packages that can:

— send messages to mobile phones

- receive messages from mobile phones

- receive messages from the Internet

— send messages to other SMS centres

- receive messages from other SMS centres.

SMS centres tend to be owned by telecommunication companies that want to offer SMS services to their customers. The software for the SMS centres is designed and developed by specialist IT companies including Logica, CMG, 


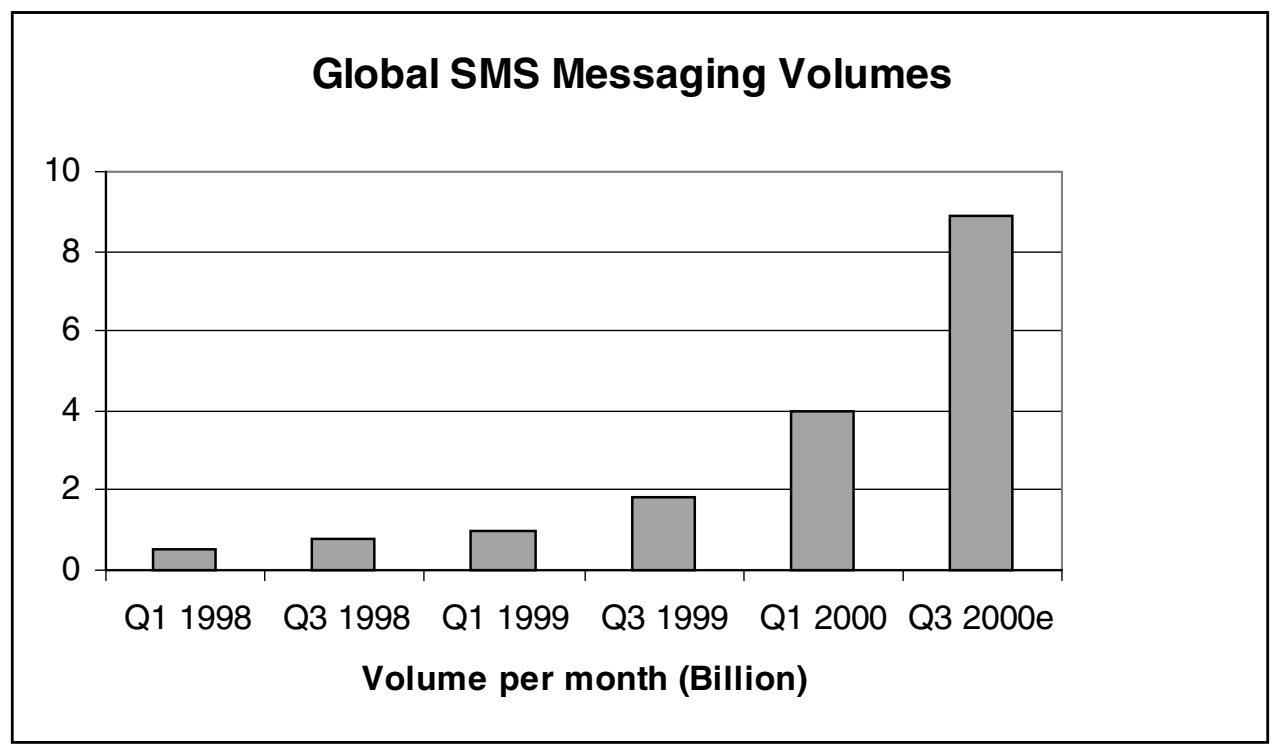

Figure 1

Source: GSM Statistics

Nokia and Sema. There is no single standard protocol for messaging systems and each of the SMS centre solution providers uses its own protocol.

The SMS business is a key part of their profitability. In fact, for some of these companies the majority of their profit growth came from their SMS divisions.

\section{THE GROWTH IN SMS}

The volume numbers for SMS services exploded in 2000. Upwards of 10 billion messages a month are sent globally using the SMS service (see Figure 1).

The key reason for the rapid growth lies in the fact that only recently has the service been truly global. Before 1998, most services were limited to the mobile operator network only. Mobile users could only send messages to other users on the same network. Clearly, that is a very limited service. Mobile operators at that time began to negotiate peering agreements that would permit the SMS centres to send messages to each other when the users were on different networks. Once these agreements were in place, the service multiplied its reach dramatically.

In addition, text messaging was associated with children and students, mainly because the cost of messaging was much cheaper than the cost of voice calls. As more people began to get messages though, especially messages alerting them to new voicemail, they began to understand the non-intrusive, but highly pertinent and time-sensitive information that could be received (and also stored in memory) using SMS.

As people became more comfortable with the service its use soared. Simple applications were deployed, including e-mail notification, information services (lottery numbers, sports scores, etc.), and other time-sensitive applications. Most of these new SMS services were deployed by the telecom operators themselves but 
the situation is changing as other organisations recognise the potential of this new communication vehicle.

\section{PRICING OF SMS SERVICES}

The mobile telecommunication operators typically charge from $2 \mathrm{p}$ to $\mathcal{E} 1$ for each message sent, with $10 \mathrm{p}$ being the average charge. This cost structure may prohibit some organisations from using SMS for marketing purposes. The pricing model will change as other organisations recognise the revenue potential of SMS and move into the market.

\section{DELIVERING SMS SERVICES}

The ability of SMS centres to send and receive messages from the Internet means that creating the necessary technical infrastructure to deliver SMS is within the capabilities of most technology-orientated organisations.

There are three key options that are being explored by the early adopters:

- developing static applications that talk to one SMS centre

- sending messages to a service provider that is connected to various SMS centres

— installing a messaging application that talks to all of the SMS centres.

There are a number of key factors that influence the choice of delivery method for the early adopters. These include:

— up-front cost

— ongoing operating costs

— flexibility of platform

- availability of internal IT resource

— quality of service desired

- volumes of messages to be sent/received.

Most have tended to opt for the service provider option and have then migrated to a messaging platform or gateway once the SMS has proved its worth.

\section{KEY FEATURES OF SMS}

Mid-1999 saw the mobile operators beginning to offer simple services over the phone. These services included:

- welcome messages

- e-mail notifications

- voice message notifications

— sports results

- lottery results.

These services were relatively straightforward and lent themselves naturally to SMS. The ability to connect directly to SMS centres via the Internet has allowed a much more extensive range of services to be developed. These new services exploit some of the beneficial features of SMS. These include their:

- personal nature: the fact that people carry a mobile phone as a personal communication device means that short text messages can be sent directly to an individual at almost any time

- near real-time delivery; a short text message can be sent to a mobile phone almost in real time, if the phone is switched on the individual will immediately be informed about the receipt of the message

- unobtrusive nature: text messages can be read when recipients want and do not therefore interrupt their current activity

- relative low cost: depending on the service provider, the cost of sending an SMS communication $\left(2 \mathrm{p}-\mathcal{f}_{1} 1\right)$ to an individual is much lower than for more traditional media such as direct mail (25-75p) or telephone marketing $\left(\delta_{1} 1.00-\delta^{2} .50\right)$ 
- simplicity: receiving and sending short text messages from a mobile phone are simple operations that do not require additional software or specialist hardware

- support for two-way communications: unlike WAP, SMS allow messages to be sent both ways

- message forwarding (ability to forward messages to peers): most SMS services allow messages to be forwarded to other individuals and, in some cases, groups of individuals

- location-based potential: the mobile technology allows the location of the phone user to be identified, opening up the potential for location-based SMS.

The key to success will be in developing SMS that are timely, relevant and pertinent.

\section{PERMISSION-BASED MARKETING}

As with e-mail, the potential for unwanted messages or SPAM is great. This will become particularly true as SMS become more widely available to third parties. The fact that most customers pay directly to retrieve text messages means that the potential impact will be even more pronounced. There is a strong move by telecommunication companies to move to a self-regulated permission-based approach to SMS.

The sanctity of the mobile phone as a personal communication tool could easily be violated by inappropriate communications. Filtering technologies are already being developed to address this issue.

\section{BUSINESS APPLICATIONS FOR SMS}

There are six main types of services (as defined by the eigroup), but that does not mean that there are not many more variations on the theme of timely, relevant information delivered directly to the user. ${ }^{2}$ The six 'SMS' types are:

- Send Me Stories

- Save Me Somehow

- Search My Server

- Sell Me Something

- Sort My Socialising

— Send Me Signals.

Send Me Stories provide a marketing message to a mobile phone user that contains information that is relevant and time sensitive, for example sending details of an in-store promotion to a loyalty card customer.

Save Me Somehow provides a marketing message to a mobile phone user that acts as coupons allowing discounts on specified goods. For example, sending details of a discount on a television to a store card customer. Or a marketing message to a mobile phone user that reminds them about an important event. For example, confirming that a large transaction has been lodged in a bank account and recommending most appropriate action.

Search My Server provides messages to customers with the objective of stimulating access to a WAP site. For example, stimulating usage of a financial information WAP site after usage has declined or service has been revamped.

Sell Me Something provides messages to customers selling a product or service and allowing them to purchase items directly through text response. For example, sending details of a new CD released by a customer's favourite band, using previous purchase or customer preference data and allowing the customer to send text message as response and initiate purchase process. As location tracking becomes widely available, the combination of time and location will prove very compelling in offering 
products or services to the consumer. Bell Mobility in the USA are currently exploring location-specific digital couponing, offering discounted products and services to subscribers within a certain radius of participating merchants. The commercial roll-out is expected in 2001. The technology will allow identification of location to within 50 feet. $^{3}$

Sort My Socialising provides messages that can be forwarded to a customer's peer group. For example, sending a customer details of a concert or webcast in a form that can be forwarded to friends and including a response mechanism that allows peers to register on WAP or website. Ensuring compatibility with the Internet messaging facilities (e-mail and ICQ), is essential if a full service offering is to be provided.

Send me Signals provides a marketing message to customers that signals an action is required. For example, a local motor dealer sends a message to a customer warning that the customer's vehicle is due a service, suggesting possible availability.

\section{LOCATION-BASED SERVICES}

The technology associated with modern cellular communications allows the location of the mobile device user to be identified to within 50 feet. This has led to the development of a number of location-based services. Although there are a number of data protection and privacy issues that will need to be addressed, a number of organisations in Europe are running pilot projects where the information is being used to drive marketing communications. If this technology becomes more widespread the application of location-based data may become the norm in some sectors, for example the automotive sector.

\section{THE IMPACT OF BLUETOOTH}

Bluetooth (a technology named after a 10th century Viking king) is being developed by Ericsson, Nokia, IBM, Toshiba and Intel to allow wireless communication between electronic devices. This technology, which will not really be available until 2002, should increase the power of SMS by facilitating more integrated communication between the mobile phone and other devices. In particular it could facilitate the payment for goods and services using the mobile phone.

\section{CONCLUSION}

SMS will grow in popularity as a marketing communication vehicle over the next few years. Its potential is only just being realised within the telecommunications industry and is still relatively unknown outside the sector. But with the creation of a global infrastructure and subsequent explosion in the use of SMS by consumers, it will not be long before other parties start to explore the opportunities that it avails. The current pricing model used by the telecommunication companies will inhibit SMS use for marketing in the short term. But the revenue potential of the SMS market will attract other providers who will increase the competition and bring down prices. The unique qualities of SMS will make it a powerful weapon in the modern marketing mix.

\section{References}

1 Bennett, M. (2000) IT Week, 2nd December.

2 'The future of SMS', Empower Interactive White Paper, 06-2000.

3 Bourrie, S. (2000) 'A sense of place: Getting there from here', Wireless Week, 15th May.

(C) Shaun Doyle 